\title{
Rapid diagnosis of IDH1-mutated gliomas by 2-HG detection with gas chromatography mass spectrometry
}

\author{
Hao Xu ${ }^{1} \cdot$ Yu-Kun Xia ${ }^{2} \cdot$ Chun-Jie Li ${ }^{2} \cdot$ Jin-Ye Zhang ${ }^{2} \cdot$ Ying Liu $^{3} \cdot$ Wei $\mathrm{Yi}^{4} \cdot$ Zhi-Yong Qin $^{1} \cdot$ Liang Chen ${ }^{1}$.

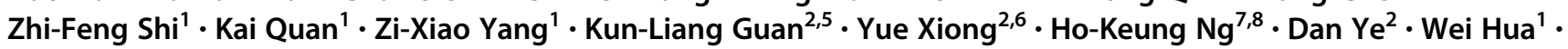 \\ Ying Mao ${ }^{1,9}$
}

Received: 1 September 2018 / Revised: 26 October 2018 / Accepted: 31 October 2018 / Published online: 20 December 2018

(c) United States \& Canadian Academy of Pathology 2018

\begin{abstract}
The metabolic genes encoding isocitrate dehydrogenase $(I D H 1,2)$ are frequently mutated in gliomas. Mutation of $I D H$ defines a distinct subtype of glioma and predicts therapeutic response. $I D H$ mutation has a remarkable neomorphic activity of converting $\alpha$-ketoglutarate $(\alpha-\mathrm{KG})$ to 2-hydroxyglutarate $(2-\mathrm{HG})$, which is now commonly referred to as an oncometabolite and biomarker for gliomas. PCR-sequencing $(n=220)$, immunohistochemistry staining (IHC, $n=220)$, and gas chromatography mass spectrometry (GC-MS, $n=87$ ) were applied to identify IDH mutation in gliomas, and the sensitivity and specificity of these strategies were compared. PCR-sequencing and IHC staining are reliable for retrospective assessment of IDHI mutation in gliomas, but both methods usually take 1-2 days, which hinders their application for rapid diagnosis. GC-MS-based methods can detect 2-HG qualitatively and quantitatively, offering information on the IDHI mutation status in gliomas with the sensitivity and specificity being $100 \%$. Further optimization of the GC-MS based methodology (so called as the mini-column method) enabled us to determine 2-HG within $40 \mathrm{~min}$ in glioma samples without complex or time-consuming preparation. Most importantly, the ratio of 2-HG/glutamic acid was shown to be a reliable parameter for determination of mutation status. The mini-column method enables rapid identification of 2-HG, providing a promising strategy for intraoperative diagnosis of $\mathrm{IDHI}$-mutated gliomas in the future.
\end{abstract}

These authors contributed equally: Hao Xu, Yu-Kun Xia, Chun-Jie Li

Supplementary information The online version of this article (https:// doi.org/10.1038/s41374-018-0163-z) contains supplementary material, which is available to authorized users.

\section{Dan Ye \\ yedan@fudan.edu.cn \\ hs_glioma@126.com \\ $\bowtie$ Ying Mao \\ maoying@fudan.edu.cn}

$\triangle$ Wei Hua

1 Department of Neurosurgery, Huashan Hospital, Fudan University, Shanghai, China

2 The Molecular and Cell Biology Lab, Institute of Biomedical Sciences, Shanghai Medical College, Fudan University, Shanghai, China

3 Department of Pathology, Shanghai Medical College, Fudan University, Shanghai, China

4 China Novartis Institutes for BioMedical Research Co. Ltd,

\section{Introduction}

Comprehensive genomic studies have revealed that somatic mutations of isocitrate dehydrogenase (IDH) frequently occur in multiple types of human cancer,

\section{Shanghai, China}

5 Department of Pharmacology and Moores Cancer Center, University of California San Diego, La Jolla, CA, USA

6 Department of Biochemistry and Biophysics, Lineberger Comprehensive Cancer Centre, University of North Carolina at Chapel Hill, Chapel Hill, NC, USA

7 Department of Anatomical and Cellular Pathology, Prince of Wales Hospital, The Chinese University of Hong Kong, Hong Kong, China

8 State Key Laboratory of Southern China in Oncology, The Chinese University of Hong Kong, Hong Kong, China

9 State Key Laboratory of Medical Neurobiology, School of Basic Medical Sciences, and The Collaborative Innovation Centre for Brain Science, Fudan University, Shanghai, China 
including a subset of gliomas [1]. Subsequent studies have shown that $I D H$ mutations may represent a powerful prognostic factor in gliomas [2]. IDHI mutation is also considered as a predictive biomarker in malignant astrocytoma for extensive resection and radio-chemotherapy to benefit patients [3]. Accordingly, IDH mutation has been introduced into the molecular subtyping and integrated diagnosis of gliomas $[4,5]$. Nearly all $I D H$ mutations target a single amino-acid residue in the enzymes' active sites, Arg132 in IDH1 and Arg172 and Arg140 in IDH2. Tumor-derived mutations in IDH1 and IDH2 abolish their normal catalytic activity in converting isocitrate to $\alpha$ ketoglutarate $(\alpha-K G)[2,6,7]$. The most remarkable feature of $I D H$ mutation is the neomorphic gain-offunction: the NADPH-dependent reduction of $\alpha-K G$ to the normally trace metabolite 2-hydroxyglutarate (2-HG), which is now commonly referred to as an oncometabolite $[6,8]$.

As a direct metabolic consequence of $I D H$ mutations, $2-\mathrm{HG}$ can be accumulated to as high as $5-35 \mu \mathrm{mol} / \mathrm{g}$ in cases of glioma harboring IDH mutations [8]. Taking advantage of the abnormally high accumulation of 2-HG, magnetic resonance spectroscopy (MRS) $[9,10]$ as well as mass spectrometry $[11,12]$ have been reported to detect 2-HG and define the $I D H$ mutation status in glioma tissues. As a non-invasive method to monitor 2HG, the sensitivity of MRS will be sharply decreased especially in small-size tumors [13]. Although the accuracy could be improved in the high field MRS (7.0 Tesla), its clinical utility is not confirmed yet [14]. Because it is considerably more sensitive than MRS, gas chromatography mass spectrometry (GC-MS)-based methodology can detect metabolites in bio-samples with a good balance of sensitivity and reliability. GC-MS was first utilized for 2-HG detection in the urine samples of aciduria patients [15], and subsequently in serum samples of AML patients harboring IDHI-R132H or IDH2R140Q mutation [16] as well as cultured cancer cell lines containing endogenous $I D H$ mutations [17]. In present study, we used GC-MS to detect 2-HG qualitatively and quantitatively, and the results offered information on the IDH1 mutation status in brain tumors with sensitivity and specificity of $100 \%$. Further optimization of the GCMS-based methodology enabled us to determine 2-HG within $40 \mathrm{~min}$. Importantly, the ratio of $2-\mathrm{HG} / \mathrm{glutamic}$ acid was utilized to detect the mutation because there was very little 2-HG among wild-type neoplasm [18, 19]. And the ratio could accurately distinguish $I D H 1$-mutated gliomas from wild-type, this may provide a new paradigm for intraoperative diagnosis and patient management in gliomas.

\section{Materials and methods}

\section{Sample acquisition and statistical analysis}

Details of patient enrollment, sample acquisition, and statistical analysis are shown in Supplementary Information. Consent forms were obtained from all patients after approval from local ethic committee in Huashan hospital. Clinical classification and grading of these tumor samples was performed by neuro-pathologists according to the 2016 WHO Classification of Tumors of the Central Nervous System [20]. Procedure for sequencing and IHC are provided in detail in Supplementary Information.

\section{GC-MS-based 2-HG detection}

Metabolite extraction was accomplished by adding $150 \mu \mathrm{L}$ of $-80{ }^{\circ} \mathrm{C}$ methanol: water mix (80\%:20\%; Cat\#34860, Sigma-Aldrich) to the brain tissue (approximately $25 \mathrm{mg}$ ) followed by $20 \mathrm{~s}$ homogenization at $4{ }^{\circ} \mathrm{C}$ (OSE-Y10, Tiangen). These chilled, methanol extracted homogenized tissues were then centrifuged at $14,000 \mathrm{rpm}$ for $6 \mathrm{~min}$ to sediment the cellular and tissue debris, and the $60 \mu \mathrm{L}$ cleared tissue supernatants were transferred to a screw-cap V-type glass-bottom vial (VAAP-31509-1232-100, CNW) and dried in a vacuum-drying apparatus (Concentrator Plus, Eppendorf) at $30^{\circ} \mathrm{C}$ for $10 \mathrm{~min}$. For analysis, the residue was mixed with $20 \mu \mathrm{L}$ N-tert-Butyldimethylsilyl-Nmethyltrifluoroacetamide (MTBSTFA, Cat\#394882, SigmaAldrich) and $35 \mu \mathrm{L}$ of pyridine (Cat\#270407, SigmaAldrich) at $70{ }^{\circ} \mathrm{C}$ for $20 \mathrm{~min}$. One microliters aliquot of derivatized sample was injected into Aligent 7890 A gas chromatography coupled with Agilent 5975C mass spectrometer. A capillary column (Cat\# 19091S-433, HP-5ms Intuvo, $30 \mathrm{~m} \times 0.25 \mathrm{~mm} \times 0.25 \mu \mathrm{m}$; Agilent Technologies) using helium as a carrier gas was utilized for separation. The parameters of GC-MS and data process are provided in Supplementary Information.

\section{GC-MS-based 2-HG quantification}

D-2-HG (Sigma-Aldrich, Cat\#94577) as a standard sample was dissolved in $\mathrm{ddH}_{2} \mathrm{O}$ at a concentration of $10 \mathrm{mM}$. Doublediluted samples were mixed with $100 \mu \mathrm{L}$ of $-80^{\circ} \mathrm{C}$ methanol: water mix (80\%:20\%), and $40 \mu \mathrm{L}$ of the mixture was transferred to a screw-cap V-type glass-bottom vial and dried in a vacuum-drying apparatus as described above, following the standard GC-MS method to detect 2-HG abundance. Accordingly, a 2-HG standard curve was made for its quantification.

For 2-HG quantification in glioma samples, metabolite extraction was accomplished by adding $200 \mu \mathrm{L}$ of $-80^{\circ} \mathrm{C}$ 

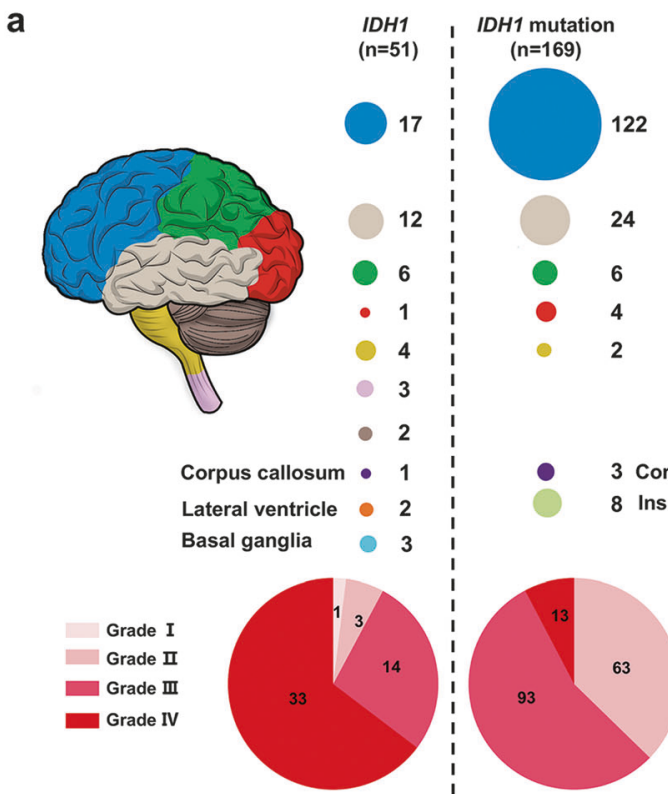

C
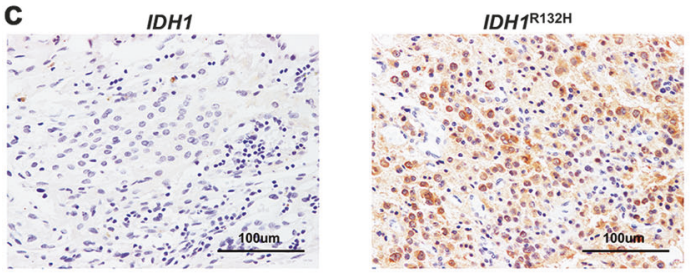

Fig. 1 Retrospective assessment of $I D H I$ mutation. a The clinical characteristic of patient population. The circle represents the population size and the color corresponds to the location. The distribution of WHO grades (lower panel) is shown. The area of each part represents the percentage contributing to the total population. A total of 220

methanol: water mix (80\%:20\%) to the brain tissue (approximately $10 \mathrm{mg}$ ) followed by $90 \mathrm{~s}$ homogenization (Tissuelyser-48, Shanghai Jingxin), and centrifugation at $14,000 \mathrm{rpm}$ for $10 \mathrm{~min}$ at $4{ }^{\circ} \mathrm{C}$. Two-hundred microliters of supernatant was transferred and dried at $30{ }^{\circ} \mathrm{C}$ for $1 \mathrm{~h}$. The residue was mixed with $35 \mu \mathrm{L}$ pyridine containing $20 \mathrm{mg} /$ $\mathrm{mL}$ Methoxyamine hydrochloride (Sigma-Aldrich, Cat\#226904) and then incubated at $70{ }^{\circ} \mathrm{C}$ for $0.5 \mathrm{~h}$. Derivatization was performed at $70{ }^{\circ} \mathrm{C}$ for $40 \mathrm{~min}$ following addition of $20 \mu \mathrm{L}$ of MTBSTFA. One-microliter aliquot of derivatized sample was injected into GC-MS for 2-HG detection and quantification.

\section{Method validation}

The mini-column method was validated according to the guidance set forth by the US-FDA for industry on bioanalytical method validation [21]. The selectivity, lower limit of quantitation (LLOQ), matrix effect, recovery, precision, accuracy, and stability were evaluated. Details and results was shown in Supplementary Information. b

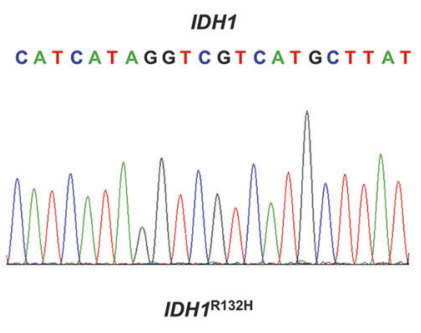

CAT CATAGG T CGT CATGCTTAT

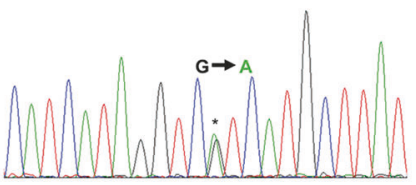

d

\begin{tabular}{|c|c|c|}
\hline \multirow[t]{2}{*}{ Method } & \multicolumn{2}{|c|}{ PCR Sequencing } \\
\hline & IDH1 mutation & $I D H 1$ \\
\hline IHC Positive & 138 & 4 \\
\hline IHC Negative & 31 & 47 \\
\hline Total & 169 & 51 \\
\hline Sensitivity (\%) & \multicolumn{2}{|c|}{81.7} \\
\hline Specificity (\%) & \multicolumn{2}{|c|}{92.2} \\
\hline
\end{tabular}

glioma samples were performed with b PCR-sequencing and $\mathbf{c}$ IHC staining to identify $I D H I^{\mathrm{R} 132 \mathrm{H}}$ mutation. Scale bars represent $100 \mu \mathrm{m}$. According to the sequencing data, the sensitivity and specificity of the IHC staining method were shown (d)

\section{Results}

\section{Retrospective assessment of IDH1 mutation by PCR-sequencing and IHC staining in gliomas}

Among 220 glioma samples, 169 heterozygous IDH1 R132H mutation were identified by PCR-sequencing (76.8\%, The WHO grades and location were shown in Fig. 1a, b). We found a much higher ratio of $\mathrm{IDH} 1$ mutation in lower grade gliomas (LGG, WHO Grades II \& III, 92.3\%) as compared to GBMs (7.7\% of IDHI mutation glioma). Moreover, IDHI-mutated gliomas were mainly located in the frontal lobe (Fig. 1a), which is in line with previous studies (for characteristic of this population, Supplementary Table S1) [22].

Next, we assessed the IDHI mutation status of glioma samples by immunohistochemistry (IHC) using an antibody specifically recognizing IDHI R132H mutant (Fig. 1c). Of $169 \mathrm{IDH} 1$-mutated samples, $138(81.7 \%)$ cases were positive for the IDHI R132H mutation by IHC (Fig. 1d). Of 51 glioma samples with wild-type IDH1, 47 (92.2\%) were confirmed by the negative staining with antibody against 

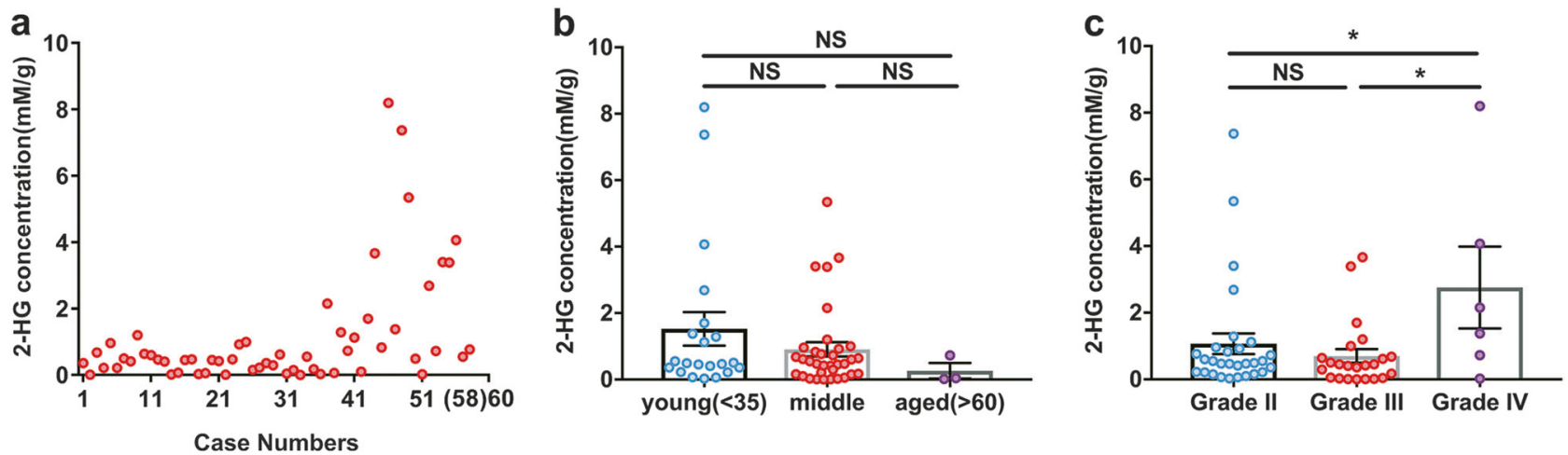

d
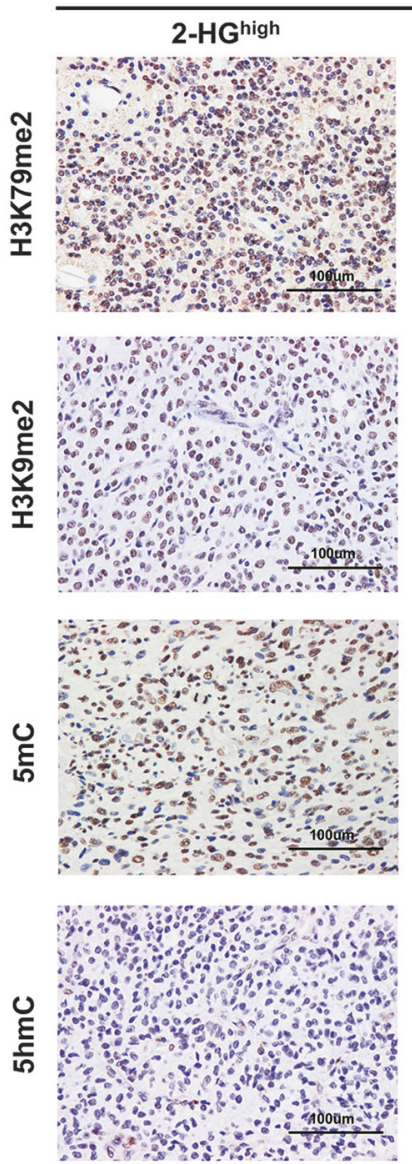

IDH1 mutation
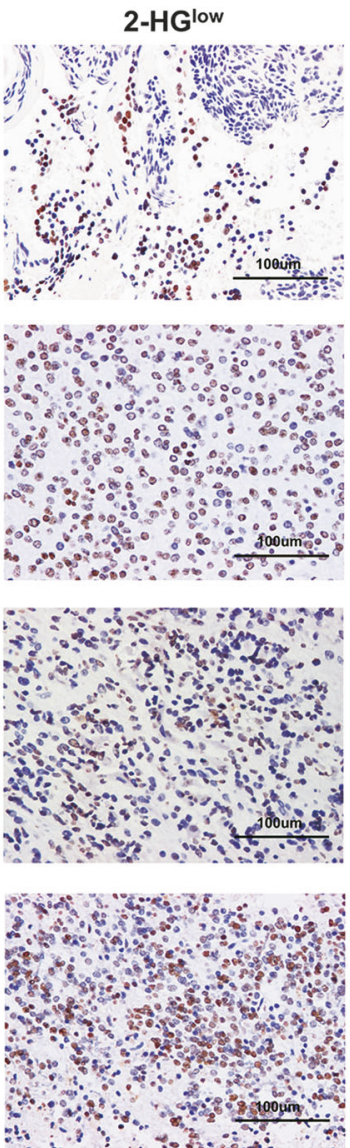
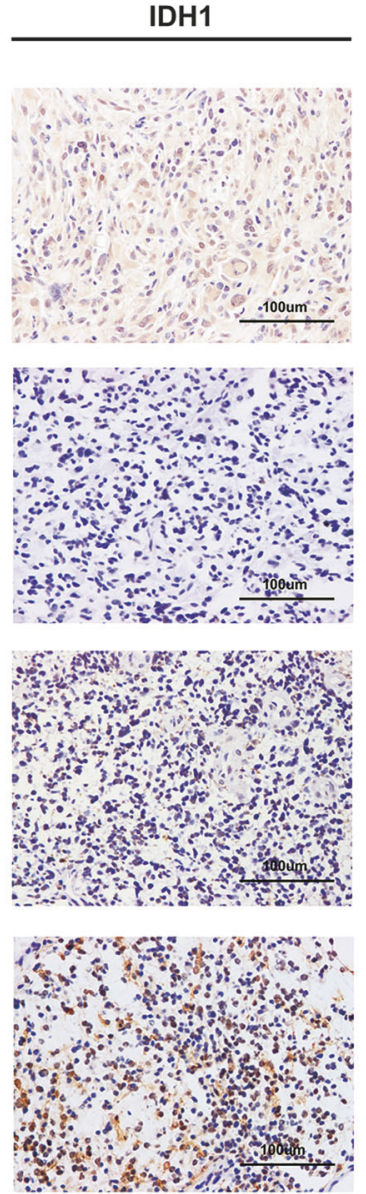
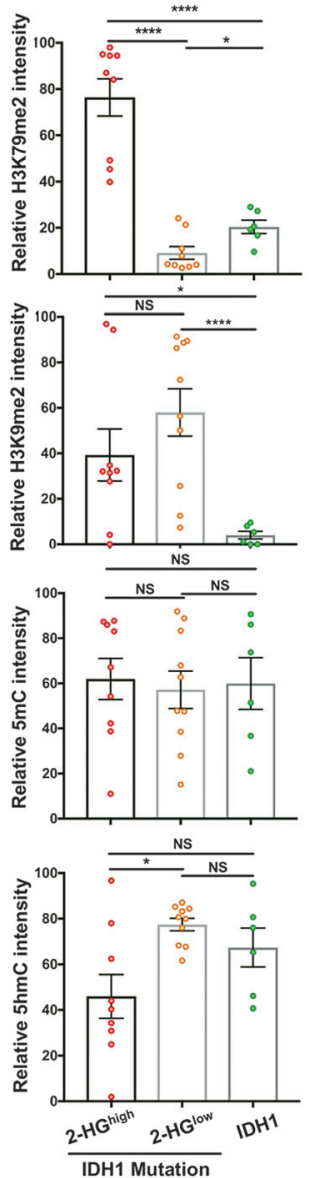

Fig. 2 Quantitative detection of 2 -HG by GC-MS in IDH1-mutated gliomas. a The concrete 2-HG concentration. b High accumulation of 2 -HG appears to be dominant in young patients (age $<35$ years), middle-aged patients ( 35 years $\leq$ age $\leq 60$ years), and $\mathbf{c}$ GBM samples. d Dose-dependent activity of $2-\mathrm{HG}$ on epigenetic regulation by IHC.

IDH1 R132H mutant, while four cases exhibited false positive IHC (Fig. 1d). With relatively high sensitivity and specificity, both PCR-sequencing and IHC staining can be used to assess the $I D H$ mutation status postoperatively in gliomas. It has to be noted that both methods usually take 1-2 days, hindering their application for rapid diagnosis.
Representative IHC data in IDH1 mutant glioma tissues (two left panels; the 2-HG ${ }^{\text {high }}$ and 2-HG ${ }^{\text {low }}$ groups) and WT tumors (right panel) are shown. Scale bars represent $100 \mu \mathrm{m}$. Furthermore, quantification of these epigenetic modification intensity is shown. Mean \pm SEM, 2-tailed $t$-test, $* P<0.05, * * P<0.01, * * * P<0.001$, $* * * * P<0.0001$

\section{Quantitative detection of 2-HG by GC-MS in IDH1-mutated gliomas}

The GC-MS technique offers a potentially sensitive and reliable assay not only for detecting 2-HG, but also for its quantification. During sample preparation of the GC-MS 
based methodology, surgically dissected glioma samples (approximately $10 \mathrm{mg}$ ) were homogenized with pre-cold methanol following centrifugation, and the supernatant was then subjected to vacuum-drying, derivatization, and GCMS detection and quantification. Standard curve was rebuilt adaptive to each detection in order to cover maximal response value within left $50 \%$ curve. In 58 cases of glioma samples harboring heterozygous IDHI R132H mutation identified by sequencing, we found that in $49 / 58(84.5 \%)$ of tested samples the 2-HG level was below $2 \mathrm{mM} / \mathrm{g}$ tissue (Fig. 2a). In a few cases harboring IDHI mutation, 2-HG concentration could reach as high as $8.2 \mathrm{mM} / \mathrm{g}$ (Fig. 2a).

The levels of 2-HG appeared to be high in young IDHImutated patients ( $<35$ years, $n=21,1.525 \pm 2.30 \mathrm{mM} / \mathrm{g}$ ) as well as middle-aged patients ( 35 years $\leq$ age $\leq 60$ years, $n=$ $34,0.909 \pm 1.22 \mathrm{mM} / \mathrm{g}$ ), and tended to be reduced in elderly patients ( $>60$ years, $n=3,0.263 \pm 0.403 \mathrm{mM} / \mathrm{g}$ ) (Fig. 2b). Due to the limited sample size of the elderly group, no statistical difference was detected (Fig. 2b). Additionally, we also observed that the 2-HG concentration was higher in GBM $(n=6,2.757 \pm 3.006 \mathrm{mM} / \mathrm{g})$, compared with LGG including WHO Grade II $(n=29,1.071 \pm 1.663 \mathrm{mM} / \mathrm{g})$ and Grade III $(n=23,0.701 \pm 0.988 \mathrm{mM} / \mathrm{g}, P<0.05)$. (Fig. $2 \mathrm{c}$ )

Altogether, these results indicate that GC-MS could provide a reliable strategy to quantify $2-\mathrm{HG}$ in gliomas harboring $I D H 1$ mutation using small amounts of tissue.

\section{Inhibition of histone demethylation by highly accumulated 2-HG in IDH1-mutated gliomas}

IDH1 R132 mutations exclusively produce D-2-HG in primary tumor tissue [8], and D-2-HG acts as an antagonist of $\alpha-\mathrm{KG}$, competitively inhibiting multiple $\alpha-\mathrm{KG} / \mathrm{Fe}(\mathrm{II})-$ dependent dioxygenases, including both histone and DNA demethylases [23, 24]. In vitro studies have shown that D-2$\mathrm{HG}$ has lowest half-maximal inhibitory concentration $\left(\mathrm{IC}_{50}\right)$ toward members of histone demethylases, ranging from 24 $\mu \mathrm{M}$ to $106 \mu \mathrm{M}$ [23]. As shown in Fig. 2a, the concentration of 2-HG (i.e., D-2-HG) ranged from $5.4 \mu \mathrm{M} / \mathrm{g}$ to $8.2 \mathrm{mM} / \mathrm{g}$ in examined $I D H I$-mutated glioma samples, suggesting the possibility that histone demethylases may be inhibited in these samples. To test this hypothesis, we analyzed histone H3 lysine 79 (H3K79) and H3 lysine 9 (H3K9) methylations in glioma samples (Fig. 2d). Our data demonstrated that H3K79 dimethylation was significantly elevated in IDH1-mutated cases in which the 2-HG level was higher than $2 \mathrm{mM} / \mathrm{g}$ neoplasm tissue (referred to as $2-\mathrm{HG}^{\text {high }}, n=$ $9)$ as compared to tumors with wild-type IDH1 $(P<0.0001$, $n=6)$. Notably, H3K79 dimethylation was not increased in IDH1-mutated glioma samples in which 2-HG level was lower than $0.1 \mathrm{mM} / \mathrm{g}$ neoplasm tissue (referred to as 2 $\mathrm{HG}^{\text {low }}, n=10$ ) when compared to tumors with wild-type IDH1. And H3K79 dimethylation was significantly higher in the $2-\mathrm{HG}^{\text {high }}$ group than the $2-\mathrm{HG}^{\text {low }}$ group $(P<0.0001)$. Methylation of H3K79 is catalyzed by DOT1L/KMT4 and plays a role in multiple cellular pathways, including telomeric silencing, cellular development, cell-cycle checkpoint, DNA repair, and regulation of transcription [25]. Our results indicate that the activity of putative H3K79 demethylase, which is yet to be identified, appears to be sensitive to the concentration of $2-\mathrm{HG}$ in cells and can only be inhibited when 2-HG is accumulated to high levels.

On the other hand, H3K9 dimethylation was significantly elevated in both the $2-\mathrm{HG}^{\text {high }}$ and $2-\mathrm{HG}^{\text {low }}$ groups harboring IDH1 mutation as compared to the IDH1 wild-type group $(P<0.05$ and $P<0.0001$, respectively). No significant change in H3K9 dimethylation levels was found between the $2-\mathrm{HG}^{\text {high }}$ and $2-\mathrm{HG}^{\text {low }}$ groups with $\mathrm{IDHI}$ mutation. Bioinformatic analysis from TCGA datasets demonstrated that most of the H3K9 dimethylation-associated histone demethylase genes [26, 27], such as $K D M 1 A, K D M 3 B$, $K D M 4 B, K D M 4 C, K D M 4 D$, KDM5B, and KDM7A, were upregulated in $I D H 1$-mutated gliomas compared to wildtype tumors (Supplementary Fig. S1). These results support the notion that $I D H 1$ mutation and 2-HG accumulation lead to inhibition of histone demethylase activity, rather than suppression of their expression.

\section{Inhibition of DNA hydroxymethylation by highly accumulated 2-HG in IDH1-mutated gliomas}

Beside $\alpha$-KG-dependent histone demethylases, 2-HG also inhibits the catalytic activity of TET1 and TET2 in vitro and in $I D H$ mutant cells $[24,28]$. In accord, $I D H$ mutation is associated with $\mathrm{CpG}$ island methylator phenotype (GCIMP) in glioma [29], and can singularly establish G-CIMP when ectopically expressed in primary astrocytes [30]. We found that the average relative intensity of 5-methylcytosine $(5 \mathrm{mC})$ did not appreciably differ among the $2-\mathrm{HG}^{\text {high }}(n=$ 9), $2-\mathrm{HG}^{\text {low }}(n=10)$, and wild-type groups $(n=6)$ (Fig. 2d). The average relative intensity of 5hydroxymethylcytosine $(5 \mathrm{hmC})$ tended to be decreased in the 2-HG ${ }^{\text {high }}$ group compared to the IDH1 wild-type group. The tendency towards reduced $5 \mathrm{hmC}$ was, however, not observed in the $2-\mathrm{HG}^{\text {low }}$ group compared to the IDH1 wildtype group. Of note, the $5 \mathrm{hmC}$ level was significantly lower in the $2-\mathrm{HG}^{\text {high }}$ group than the $2-\mathrm{HG}^{\text {low }}$ group $(P<0.05)$. Gene expression data from TCGA demonstrated that all there TET genes were significantly upregulated in IDHImutated gliomas compared to IDH1 wild-type tumors (Supplementary Fig. S2), supporting the notion that $I D H 1$ mutation and 2-HG lead to inhibition of TET activity in gliomas, rather than suppression of their expression. Additionally, these results also suggest that the catalytic activity of TET enzymes can only be inhibited when 2-HG is accumulated to high levels in $I D H$-mutated gliomas. 
a
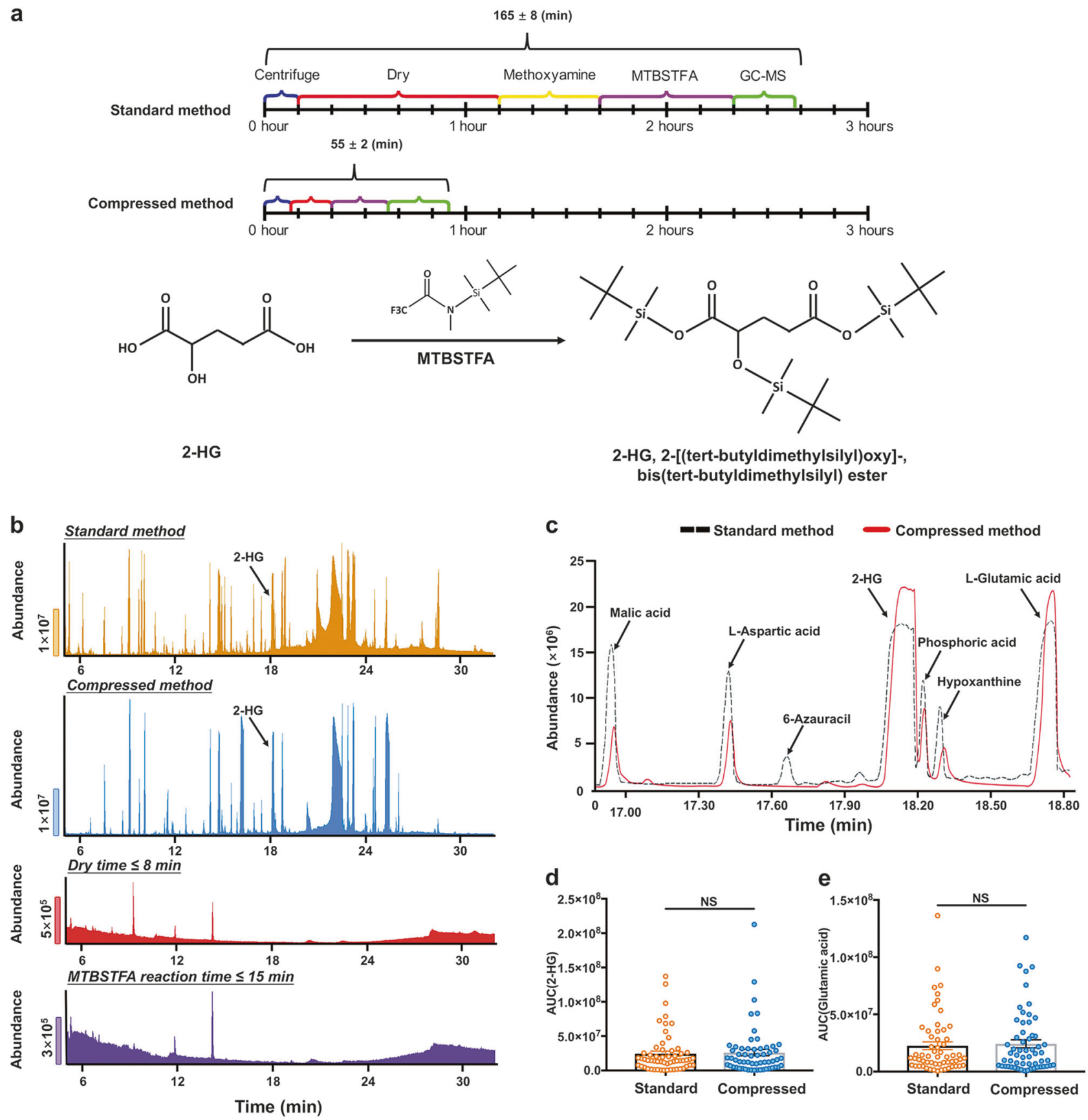

Fig. 3 A compressed GC-MS method for rapid 2-HG detection. a Diagram showing the standard and compressed methods. b Metabolite profiling in $I D H 1$-mutated glioma samples. Arrow pointing the 2-HG peak. When the vacuum-drying time is shortened to $8 \mathrm{~min}$ or the MTBSTFA reaction time is cut to $15 \mathrm{~min}$, the 2-HG peak cannot be

\section{A compressed GC-MS procedure for rapid 2-HG detection}

Both PCR-sequencing and IHC staining methods require usually more than 1 day to complete, making them not applicable for rapid diagnosis of $I D H$ mutation in clinical practice. The standard procedure for GC-MS method

detected by GC-MS. c Comparison of metabolite profiles determined by the standard and compressed methods. The AUC of $\mathbf{d} 2-\mathrm{HG}$ and e L-glutamic acid in IDH1-mutated glioma samples $(n=58)$ as determined by the standard and compressed GC-MS methods

includes homogenization, centrifugation, vacuum-drying, oximation by methoxyamine hydrochloride (MOX), and derivatization by N-tert-Butyldimethylsilyl-N- methyltrifluoroacetamide (MTBSTFA), which generally takes about $3 \mathrm{~h}$ (i.e., $165 \pm 8 \mathrm{~min}$ ) (Fig. $3 \mathrm{a}$ ). Of note, $2-\mathrm{HG}$ is a small molecular metabolite without carbonyl groups and thus cannot react with MOX [31]. Considering that 2-HG 

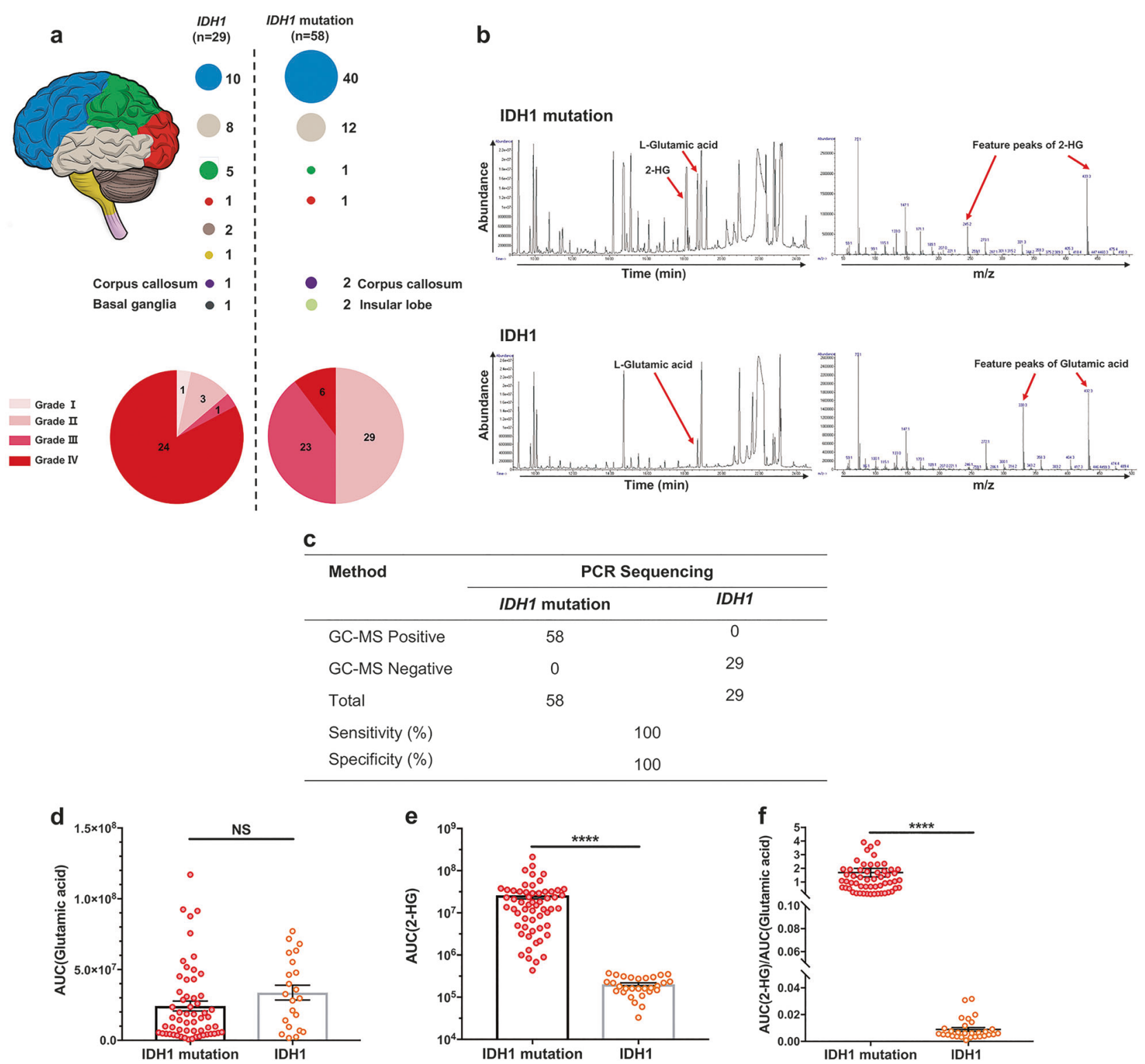

Fig. 4 The compressed method can commendably predict IDHI mutation status. a The clinical characteristic of patient population. The circle represents the population size and the color corresponds to the location. The distribution of WHO grades (lower panel) is shown. b Metabolite profiling in $I D H I$-mutated or WT glioma samples. c The

sensitivity and specificity of the method. The AUC of $\mathbf{d}$ L-glutamic acid and e 2-HG in IDH1-mutated or WT glioma samples was determined by the compressed method. For 2-HG standardization, f the ratio of $2-\mathrm{HG} / \mathrm{L}-$ glutamic acid is calculated and shown

contains three hydroxyls and thereby can react with MTBSTFA (Fig. 3a), we developed a compressed GC-MS procedure with a goal to detect 2-HG rapidly. To accelerate the GC-MS procedure, we tested various conditions to shorten the time-period for each step of GC-MS and their combinations. We determined that the centrifugation time can be reduced to as short as $6 \mathrm{~min}$, the vacuumdrying time to as short as $10 \mathrm{~min}$, the oximation step by methoxyamine hydrochloride can be omitted, and the MTBSTFA reaction time can be cut to $20 \mathrm{~min}$ without losing the detection of 2-HG peak (Fig. 3a, b). As such, the compressed GC-MS procedure can be completed from tissue sample preparation to $2-\mathrm{HG}$ detection in less than an hour $(55 \pm 2 \mathrm{~min})$.

Next, we tested the potential for clinical utility of this compressed GC-MS based 2-HG detection method in a cohort of 87 glioma patients (for characteristic of this population, Supplementary Table S2). The workflow of operation starts at the diagnosis based on MRI before surgery and ends at retrospective assessment of $\mathrm{IDHI}$ mutation by PCR-sequencing and IHC staining. Surgically dissected gliomas samples were directly subjected to metabolite profiling by using the GC-MS based methods (Supplementary Fig. S3). The compressed GC-MS 
a

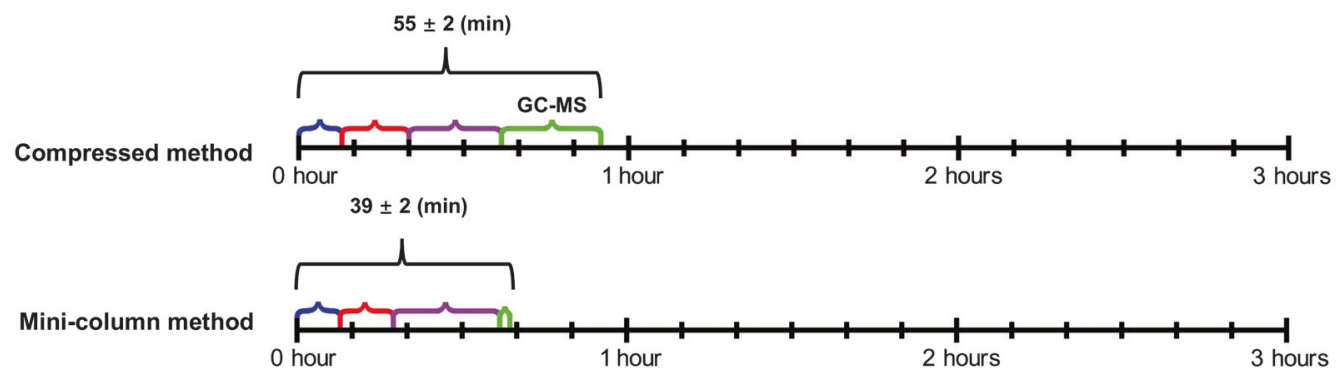

b
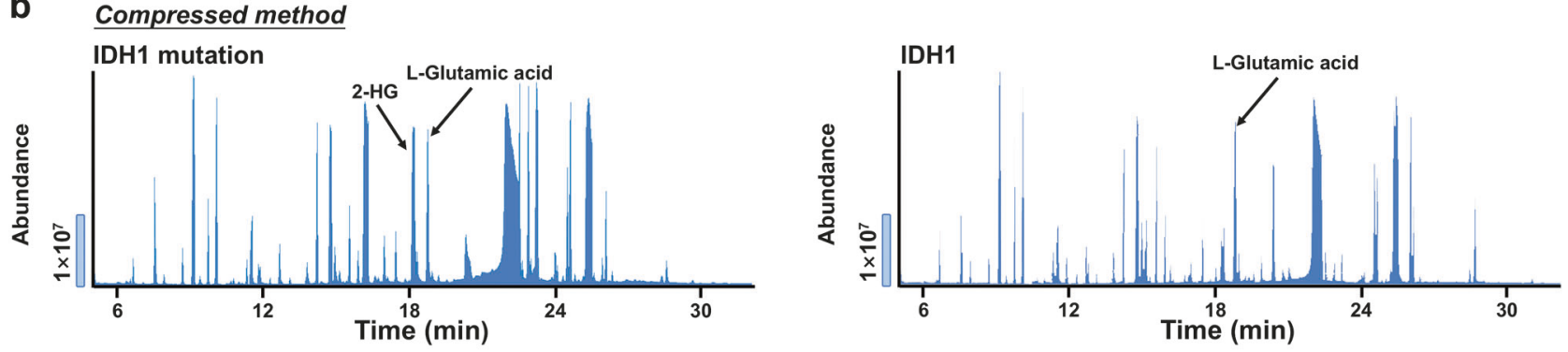

Mini-column method
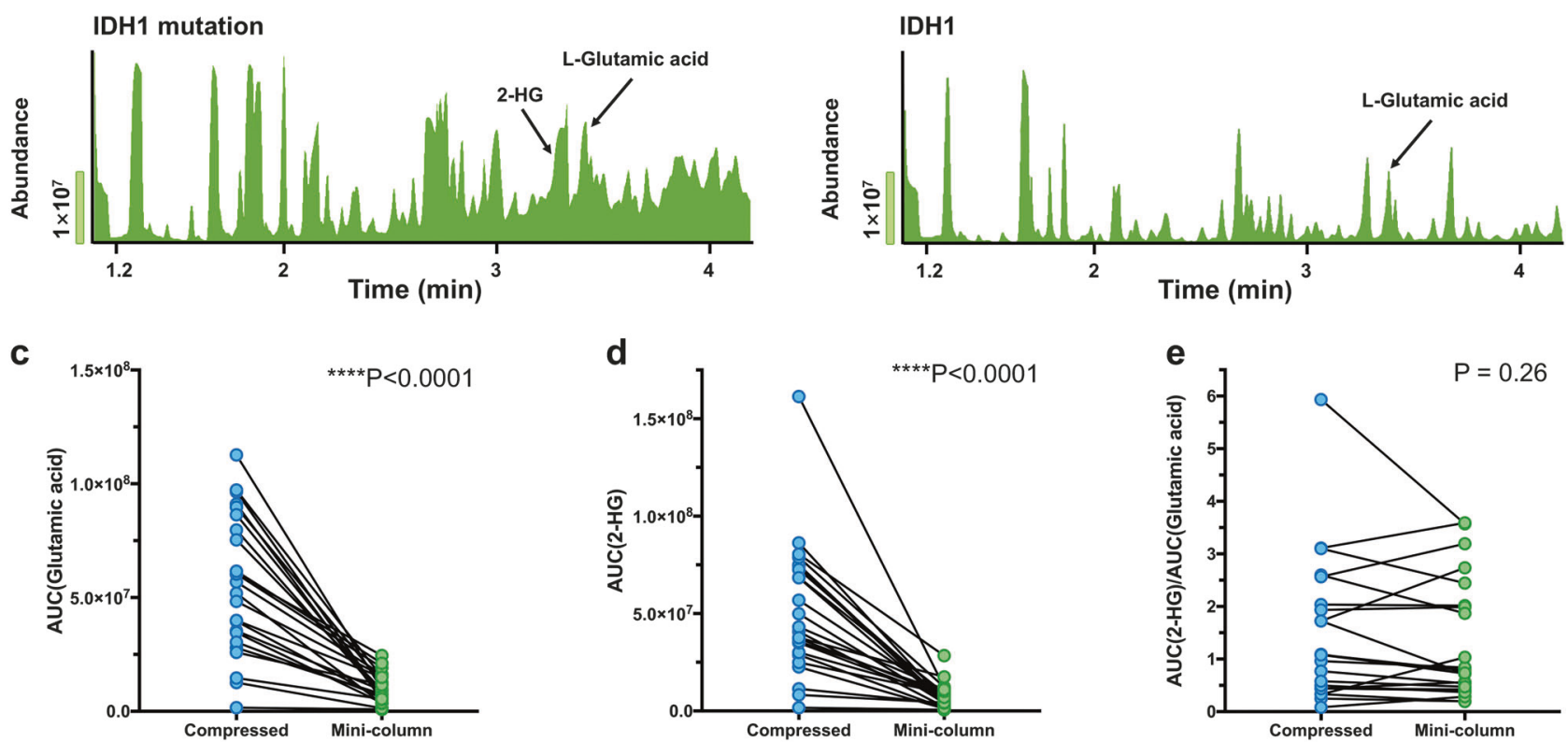

Fig. 5 A mini-column method for rapid diagnosis. a The GC-MS running time was shortened dramatically by cutting down the GC capillary column and total detection time was within $40 \mathrm{~min}$. b Metabolite profiling in IDH1-mutated glioma samples. The AUC of

method omits the MOX oximation step and interferes the detection of metabolites containing carbonyl groups. Indeed, the AUC (area under curve) of metabolites with carbonyl groups, such as 6-azauracil and hypoxanthine (Fig. 3c), determined by the compressed GC-MS method was clearly smaller than that determined by the standard GC-MS method. Neither 2-HG nor L-glutamic acid, which is commonly used for $2-\mathrm{HG}$ standardization $[32,33]$, has carbonyl groups. Accordingly, the AUC of 2-HG or L-glutamic acid determined by the compressed c L-glutamic acid $\mathbf{d} 2-\mathrm{HG}$, and $\mathbf{e}$ the ratio of $2-\mathrm{HG} / \mathrm{L}$-glutamic acid in 24 cases of IDH1-mutated glioma samples was determined by the compressed and mini-column methods

GC-MS method was similar to that analyzed by the standard method ( $n=58$, paired $t$-test; Fig. $3 \mathrm{~d}$, e).

Among these 87 cases, 58 samples were identified to harbor IDH1 mutation by PCR-sequencing (Supplementary Table S2). By applying the compressed GC-MS method, we detected 2-HG accumulation in all $58 \mathrm{IDH}$-mutated samples (Fig. 4a, b, c). None of the examined 29 samples, which had been identified to contain wild-type IDH1 by PCR-sequencing, showed 2-HG accumulation when analyzed by the compressed GC-MS method (Fig. 4b). Thus, 
these findings indicate a $100 \%$ accuracy of the compressed GC-MS method in identifying $I D H$-mutated glioma samples. Consistent with our earlier finding (Fig. 1a), we found that IDHI-mutated/2-HG-positive cases were enriched in WHO Grade II and III gliomas and mainly located in the frontal lobe by using the compressed method (Fig. 4a). The AUC of L-glutamic acid determined by the compressed GCMS remains constant between IDHI-mutated and wild-type tumors (Fig. 4d). This, together with the close retention time of L-glutamic acid (GLU, $18.72 \mathrm{~min}$ ) and 2-HG (18.12 min), provides a convenient and semi-quantitative internal control to the rapid GC-MS procedure. We found that the ratio of 2HG/GLU was significantly increased in all cases of IDHImutated cases $(1.685 \pm 2.367, \quad n=58 ; \quad P<0.0001)$ compared to the IDH1 wild-type samples $(0.0096 \pm 0.0078$, $n=29$, Fig. 4 d, e, f). The ratio of $2-\mathrm{HG} / \mathrm{GLU}$, therefore, represents a more accurate measurement to discriminate between $I D H I$ mutant and wild-type gliomas: a ratio of 2HG/GLU below 0.04 indicated wild-type IDH status, a ratio above 0.1 was only found in tumors with $I D H 1$ mutation, and ratios in the range of 0.04 to 0.1 were not seen in two cohorts of the analyzed samples (Fig. 4f).

\section{A faster and qualitative GC-MS procedure for 2-HG detection and rapid diagnosis of IDH1-mutated gliomas}

To get a rapid diagnosis of $I D H$ mutation, the capillary column in the GC machine was cut down from 30 to 5 meters (minicolumn method). As a result, the GC running time was dramatically shortened from $32 \mathrm{~min}$ (standard method) to $4.2 \mathrm{~min}$ (mini-column method). During GC running period, the 2-HG peaks appear at $18.1 \mathrm{~min}$ and $3.3 \mathrm{~min}$ when the standard and mini-column methods are applied, respectively. Total detection time (mini-column method) was $39 \pm 2$ min compared with $55 \pm 2 \mathrm{~min}$ of the compressed method $(P<0.0001$, Fig. 5a) (Supplementary Table S3). The separation efficiency and peaks deceased obviously using 5-meter column method, but the 2HG peak can still be sufficiently separated by this mini-column and clearly identified by MS, appearing at $3.3 \mathrm{~min}$ instead of $18.1 \mathrm{~min}$ on the standard column (Fig. 5b).

To evaluate the accuracy and clinical utility of the minicolumn method in detecting 2-HG and identifying $I D H$ mutation status in tumor tissue, a cohort of 43 surgically dissected gliomas were subjected to 2-HG detection by both the compressed method and mini-column method. Of these 43 samples, 24 harbor $I D H 1$ mutation as determined by PCR-sequencing (data not shown), and all these 24 samples were 2-HG positive as determined by either the compressed or mini-column GC-MS method. The AUC of L-glutamic acid or 2-HG was significantly smaller on the mini-column method $(P<0.001$, paired $t$-test; Fig. $5 \mathrm{c}, \mathrm{d})$. Nevertheless, the ratio of $2-\mathrm{HG} / \mathrm{GLU}$ determined by the mini-column method was similar to that determined by the compressed GC-MS procedure ( $P=0.26$, paired $t$-test; Fig. 5 e). Furthermore, the ratio of $2-\mathrm{HG} / \mathrm{GLU}$ is commonly above 0.1 in all the examined 24 samples harboring $I D H 1$ mutation as determined by the mini-column method, while the ratio is $<$ 0.04 in 19 wild-type samples, suggesting that the 2-HG/ GLU ratio may represent as a reliable index to judge the IDH mutation status in clinical cases (Supplementary Fig. S4). Collectively, these results indicate that the minicolumn method, although not suitable for quantification, can reliably and rapidly identify 2-HG and thus IDHI mutation status in surgically dissected glioma samples, providing a novel method with potentially broad clinical utility for rapid diagnosis of $I D H$ mutation.

\section{Discussion}

IDH mutations define distinct subtypes within otherwise heterogeneous gliomas [34, 35], and these subgroups exhibit unique clinical features and response to therapy [3, 36, 37]. Precise diagnosis of $I D H$ mutation is critical for patient care management in gliomas. In recent years, rapid progress has been made in the development of mass spectrometry technology, including the identification of small metabolites. The discovery of D-2-HG production by mutant $I D H$ provides an excellent example to elucidate the promise of the mass spectrometry-based technology [8].

In this study, we developed GC-MS based methods for 2HG detection and quantification. Our data demonstrate that D2-HG concentration varies among IDHI-mutated gliomas, ranging from $5.4 \mu \mathrm{M} / \mathrm{g}$ to $8.2 \mathrm{mM} / \mathrm{g}$ neoplasm tissue. $2-\mathrm{HG}$ carries an asymmetric carbon atom in its carbon backbone, leading to the formation of two enantiomers: D-2-HG and L-2HG. Both enantiomers of 2-HG are normal endogenous metabolites but may differ in the potency to bind and inhibit the activity of $\alpha$-KG-dependent dioxygenases. The activity of D-2HG to inhibit $\alpha$-KG-dependent dioxygenases including histone and DNA demethylases has been well recognized. Comprehensive in vitro studies have reported that the activity of D-2HG to inhibit members of the $\alpha$-KG-dependent dioxygenase family varies greatly, with histone demethylases KDM4A/ JMJD2A and KDM4C/JMJD2C being the most sensitive $\left(\mathrm{IC}_{50}=24 \mu \mathrm{M}\right.$ and $79 \mu \mathrm{M}$, respectively), and proline hydroxylase domain protein 2 (PHD2) and $\gamma$-butyrobetaine hydroxylase (BBOX) being very resistant $\left(\mathrm{IC}_{50}=7.3 \mathrm{mM}\right.$ and 13.2 $\mathrm{mM}$, respectively) [23]. Additionally, D-2-HG also inhibits mouse Tet 1 and Tet 2 in vitro $\left(\mathrm{IC}_{50}=4 \mathrm{mM}\right.$ and $5 \mathrm{mM}$, respectively) [28, 38]. Therefore, $\alpha$-KG-dependent dioxygenases are predicted to be differently inhibited by D-2-HG in glioma harboring $\mathrm{IDH}$ mutation, and ones with the lowest $\mathrm{IC}_{50}$ value for D-2-HG will be preferentially inhibited first. Supporting this notion, we show in this study that 2-HG 
accumulation in both the $2-\mathrm{HG}^{\text {low }}$ and $2-\mathrm{HG}^{\text {high }}$ groups are sufficient to inhibit $\alpha$-KG-dependent KDM4A/JMJD2A and $\mathrm{KDM} 4 \mathrm{C} / \mathrm{JMJD} 2 \mathrm{C}$ which are highly susceptible to 2-HG inhibition [23]. On the other hands, 2-HG accumulated in the 2-HG ${ }^{\text {low }}$ group of gliomas is not sufficient to effectively inhibit TETs, and higher accumulation of 2-HG (e.g., 4 to $5 \mathrm{mM}$ ) [38] will be needed for catalytic inhibition of TET. Collectively, these results provide in vivo evidence re-affirming inhibition of $\alpha$-KG-dependent histone and DNA demethylases by highly accumulated 2-HG in IDHI-mutated gliomas. Clinical implications of the observed differential inhibition of diverse $\alpha$-KGdependent dioxygenases needs further investigation.

Rapid diagnosis of $I D H$ mutation is urgently needed to help neurosurgeons to design strategies for treating brain tumors for several reasons. First, $I D H$ mutation can distinguish LGG from gliosis and normal brain tissues. Second, 2-HG concentration usually sharply falls among the margins of gliomas [12], which is very helpful for recognizing the tumor boundary. Third, rapid diagnosis of IDH mutation would have potentially significant benefit for deciding the extent of resection. If the tumor is located in non-functional areas of the brain, total resection or maximal surgical resection will definitely benefit patients harboring $I D H 1$ mutations. If the $I D H$-mutated tumor is next to or located in functional areas of the brain, these tumors should be left to preserve critical neurological functions and are supposed to be highly sensitive to radio-chemotherapy [36]. In the current study, we have developed a new compressed GC-MSbased method, which can accurately and rapidly identify IDH mutation status in brain tumors by monitoring 2-HG. After further modification, the GC running time has been successfully shortened to $4.2 \mathrm{~min}$, and thus enables us to detect 2-HG in small amounts of glioma samples within 40 min after surgery. Very recently, desorption electrospray ionization-mass spectrometry (DESI-MS) was reported to identify 2-HG (around $3 \mathrm{~min}$, regardless of the sample preparation time) in frozen tissue smears and help the rapid molecular diagnosis of $I D H$ mutation in gliomas [39]. Both DESI-MS and GC-MS are convenient for 2-HG detection, because DESI-MS doesn't need a reference library and GCMS owns a standard Mass Database, which can be widely applied to establish a standard protocol for detection of metabolites including 2-HG and any other oncometabolites [40]. It has to be noted that establishment of the reference library is needed before conducting the DESI-MS to determine tumor cell percentage(TCP) [41]. Liquid chromatography/electrospray ionization tandem mass spectrometry (LC/ESI-MS/MS) method was developed to accurately detect 2-HG [42], while it couldnot completely distinguish the mutated from the wild-type. Because there is little 2-HG among wild-type tumor, accurate diagnosis couldnot be made solely dependent on 2-HG. We believe that both ambient ionization methods and GC-MS-based methods will have broad clinical utility by helping to intraoperatively diagnose $I D H$ mutation status, expedite clinical decision-making, and improve the care and treatment of neuro-oncologic patients in the near future.

Acknowledgements We extremely thank R. Graham Cooks for his suggestion and manuscript revise. We thank all of the surgeons (Department of Neurosurgery, Huashan Hospital, Fudan University) for their effort in sample collection. Also, we thank members of the Fudan MCB laboratory for discussion and support throughout this study. This work was supported by the National Key R\&D Program of China (No. 2016YFA0501800), NSFC grants (No. 81572483, 81522033, and 81372198), the Shanghai Committee of Science and Technology, China (No. 16JC1404000, No. 15441904500) and Natural Science Foundation and Major Basic Research Program of Shanghai, China (No. 16JC1420100).

\section{Compliance with ethical standards}

Conflict of interest The authors declare that they have no conflict of interest.

\section{References}

1. Parsons DW, Jones S, Zhang X, et al. An integrated genomic analysis of human glioblastoma multiforme. Science. 2008;321:1807-12.

2. Yan H, Parsons DW, Jin G, et al. IDH1 and IDH2 mutations in gliomas. N Engl J Med. 2009;360:765-73.

3. Beiko J, Suki D, Hess KR, et al. IDH1 mutant malignant astrocytomas are more amenable to surgical resection and have a survival benefit associated with maximal surgical resection. Neuro Oncol. 2014;16:81-91.

4. Ceccarelli M, Barthel FP, Malta TM, et al. Molecular profiling reveals biologically discrete subsets and pathways of progression in diffuse glioma. Cell. 2016;164:550-63.

5. Eckel-Passow JE, Lachance DH, Molinaro AM, et al. Glioma groups based on $1 \mathrm{p} / 19 \mathrm{q}, \mathrm{IDH}$, and TERT promoter mutations in tumors. N Engl J Med. 2015;372:2499-508.

6. Ward PS, Patel J, Wise DR, et al. The common feature of leukemia-associated IDH1 and IDH2 mutations is a neomorphic enzyme activity converting alpha-ketoglutarate to 2hydroxyglutarate. Cancer Cell. 2010;17:225-34.

7. Zhao $\mathrm{S}$, Lin $\mathrm{Y}, \mathrm{Xu} \mathrm{W}$, et al. Glioma-derived mutations in IDH1 dominantly inhibit IDH1 catalytic activity and induce HIF-1alpha. Science. 2009;324:261-5.

8. Dang L, White DW, Gross S, et al. Cancer-associated IDH1 mutations produce 2-hydroxyglutarate. Nature. 2009;462:739-44.

9. Andronesi OC, Kim GS, Gerstner E, et al. Detection of 2hydroxyglutarate in IDH-mutated glioma patients by in vivo spectral-editing and 2D correlation magnetic resonance spectroscopy. Sci Transl Med. 2012;4:116ra4.

10. Choi C, Ganji SK, DeBerardinis RJ, et al. 2-hydroxyglutarate detection by magnetic resonance spectroscopy in IDH-mutated patients with gliomas. Nat Med. 2012;18:624-9.

11. Ohka F, Ito M, Ranjit M, et al. Quantitative metabolome analysis profiles activation of glutaminolysis in glioma with IDH1 mutation. Tumour Biol. 2014;35:5911-20.

12. Santagata S, Eberlin LS, Norton I, et al. Intraoperative mass spectrometry mapping of an onco-metabolite to guide brain tumor surgery. Proc Natl Acad Sci USA. 2014;111:11121-6.

13. de la Fuente MI, Young RJ, Rubel J, et al. Integration of 2hydroxyglutarate-proton magnetic resonance spectroscopy into 
clinical practice for disease monitoring in isocitrate dehydrogenase-mutant glioma. Neuro Oncol. 2016;18:283-90.

14. Andronesi OC, Loebel F, Bogner W, et al. Treatment response assessment in IDH-mutant glioma patients by noninvasive 3D functional spectroscopic mapping of 2-hydroxyglutarate. Clin Cancer Res. 2016;22:1632-41.

15. Struys EA, Verhoeven NM, Roos B, Jakobs C. Disease-related metabolites in culture medium of fibroblasts from patients with D2-hydroxyglutaric aciduria, L-2-hydroxyglutaric aciduria, and combined D/L-2-hydroxyglutaric aciduria. Clin Chem. 2003;49:1133-8.

16. Sellner L, Capper D, Meyer J, et al. Increased levels of 2hydroxyglutarate in AML patients with IDH1-R132H and IDH2R140Q mutations. Eur J Haematol. 2010;85:457-9.

17. Ma S, Jiang B, Deng W, et al. D-2-hydroxyglutarate is essential for maintaining oncogenic property of mutant IDH-containing cancer cells but dispensable for cell growth. Oncotarget. 2015;6:8606-20.

18. Ye D, Guan KL, Xiong Y. Metabolism, activity, and targeting of D- and L-2-hydroxyglutarates. Trends Cancer. 2018;4:151-65.

19. Colvin H, Nishida N, Konno M, et al. Oncometabolite D-2hydroxyglurate directly induces epithelial-mesenchymal transition and is associated with distant metastasis in colorectal cancer. Sci Rep. 2016;6:36289.

20. Louis DN, Perry A, Reifenberger G, et al. The 2016 World Health Organization classification of tumors of the central nervous system: a summary. Acta Neuropathol. 2016;131:803-20.

21. US Food and Drug Administration, Guidance for Industry: Bioanalytical Method Validation, 2001, [Accessed 15 November 2017]. Available from http://www.fda.gov/downloads/Drugs/ Guidance/ucm070107.pdf.

22. Ichimura K, Pearson DM, Kocialkowski S, et al. IDH1 mutations are present in the majority of common adult gliomas but rare in primary glioblastomas. Neuro Oncol. 2009;11:341-7.

23. Chowdhury R, Yeoh KK, Tian YM, et al. The oncometabolite 2hydroxyglutarate inhibits histone lysine demethylases. EMBO Rep. 2011;12:463-9.

24. Xu W, Yang H, Liu Y, et al. Oncometabolite 2-hydroxyglutarate is a competitive inhibitor of alpha-ketoglutarate-dependent dioxygenases. Cancer Cell. 2011;19:17-30.

25. Farooq Z, Banday S, Pandita TK, Altaf M. The many faces of histone H3K79 methylation. Mutat Res Rev Mutat Res. 2016;768:46-52.

26. Cerami E, Gao J, Dogrusoz U, et al. The cBio cancer genomics portal: an open platform for exploring multidimensional cancer genomics data. Cancer Discov. 2012;2:401-4.

27. Gao J, Aksoy BA, Dogrusoz U, et al. Integrative analysis of complex cancer genomics and clinical profiles using the cBioPortal. Sci Signal. 2013;6:pl1.
28. Koivunen P, Lee S, Duncan CG, et al. Transformation by the (R)enantiomer of 2-hydroxyglutarate linked to EGLN activation. Nature. 2012;483:484-8.

29. Noushmehr H, Weisenberger DJ, Diefes K, et al. Identification of a $\mathrm{CpG}$ island methylator phenotype that defines a distinct subgroup of glioma. Cancer Cell. 2010;17:510-22.

30. Turcan S, Rohle D, Goenka A, et al. IDH1 mutation is sufficient to establish the glioma hypermethylator phenotype. Nature. 2012;483:479-83.

31. Yan Y, Rempel DL, Holy TE, Gross ML. Mass spectrometry combinations for structural characterization of sulfated-steroid metabolites. J Am Soc Mass Spectrom. 2014;25:869-79.

32. Figueroa ME, Abdel-Wahab O, Lu C, et al. Leukemic IDH1 and IDH2 mutations result in a hypermethylation phenotype, disrupt TET2 function, and impair hematopoietic differentiation. Cancer Cell. 2010;18:553-67.

33. Sahm F, Capper D, Pusch S, et al. Detection of 2-hydroxyglutarate in formalin-fixed paraffin-embedded glioma specimens by gas chromatography/mass spectrometry. Brain Pathol. 2012;22:26-31.

34. Sturm D, Witt H, Hovestadt V, et al. Hotspot mutations in H3F3A and IDH1 define distinct epigenetic and biological subgroups of glioblastoma. Cancer Cell. 2012;22:425-37.

35. Yang H, Ye D, Guan KL, Xiong Y. IDH1 and IDH2 mutations in tumorigenesis: mechanistic insights and clinical perspectives. Clin Cancer Res. 2012;18:5562-71.

36. Buckner JC, Shaw EG, Pugh SL, et al. Radiation plus procarbazine, CCNU, and vincristine in low-grade glioma. N Engl J Med. 2016;374:1344-55.

37. Kawaguchi T, Sonoda Y, Shibahara I, et al. Impact of gross total resection in patients with WHO grade III glioma harboring the IDH $1 / 2$ mutation without the $1 \mathrm{p} / 19 \mathrm{q}$ co-deletion. J Neurooncol. 2016;129:505-14.

38. Laukka T, Mariani CJ, Ihantola T, et al. Fumarate and succinate regulate expression of hypoxia-inducible genes via TET Enzymes. J Biol Chem. 2016;291:4256-65.

39. Pirro V, Alfaro CM, Jarmusch AK, et al. Intraoperative assessment of tumor margins during glioma resection by desorption electrospray ionization-mass spectrometry. Proc Natl Acad Sci USA. 2017;114:6700-5.

40. Jarmusch AK, Pirro V, Baird Z, et al. Lipid and metabolite profiles of human brain tumors by desorption electrospray ionizationMS. Proc Natl Acad Sci USA. 2016;113:1486-91.

41. Pirro V, Jarmusch AK, Alfaro CM, et al. Utility of neurological smears for intrasurgical brain cancer diagnostics and tumour cell percentage by DESI-MS. Analyst. 2017;142:449-54.

42. Kanamori M, Maekawa M, Shibahara I, et al. Rapid detection of mutation in isocitrate dehydrogenase 1 and 2 genes using mass spectrometry. Brain Tumor Pathol. 2018;35:90-6. 